\title{
INTEGRAL OPERATORS ON SPACES OF CONTINUOUS VECTOR-VALUED FUNCTIONS
}

\author{
PAULETTE SAAB
}

(Communicated by William J. Davis)

\begin{abstract}
Let $X$ be a compact Hausdorff space, let $E$ be a Banach space, and let $C(X, E)$ stand for the Banach space of $E$-valued continuous functions on $X$ under the uniform norm. In this paper we characterize integral operators (in the sense of Grothendieck) on $C(X, E)$ spaces in terms of their representing vector measures. This is then used to give some applications to nuclear operators on $C(X, E)$ spaces.
\end{abstract}

\section{INTRODUCTION}

Let $X$ be a compact Hausdorff space; let $E$ and $F$ be Banach spaces. Denote by $C(X, E)$ the space of all continuous $E$-valued functions defined on $X$ under the uniform norm. C. Swartz showed [9] that a bounded linear operator $T: C(X, E) \rightarrow F$ with representing measure $G$ is absolutely summing if and only if each of the values of $G$ is an absolutely summing operator from $E$ to $F$ and $G$ is of bounded variation as a measure taking its values in the space of absolutely summing operators from $E$ to $F$ equipped with the absolutely summing norm. In this paper we shall extend Swartz's result to the class of (Grothendieck) integral operators on $C(X, E)$ spaces. More precisely, we shall show that a bounded linear operator $T: C(X, E) \rightarrow F$ with representing measure $G$ is an integral operator if and only if each of the values of $G$ is an integral operator from $E$ to $F$ and $G$ is of bounded variation as a vector measure taking its values in the space of integral operators from $E$ to $F$ equipped with the integral norm. This result is then used to give some applications to nuclear operators on $C(X, E)$ spaces.

\section{Preliminaries}

If $X$ is a compact Hausdorff space and $E$ is a Banach space, then $C(X, E)$ will denote the Banach space of all continuous $E$-valued functions equipped with the uniform norm. It is well known [4, p. 182] that the dual of $C(X, E)$

Received by the editors August 8, 1989 and, in revised form, January 12, 1990.

1980 Mathematics Subject Classification (1985 Revision). Primary 46E40, 46G10; Secondary 28B05, 28B20.

Supported in part by NSF Grant DMS-87500750. 
is isometrically isomorphic to the space $M\left(X, E^{*}\right)$ of all regular $E^{*}$-valued measures on $X$ that are of bounded variation. When $E$ is the scalar field, we will simply write $C(X)$ and $M(X)$ for $C(X, E)$ and $M\left(X, E^{*}\right)$. If $\mu \in$ $M\left(X, E^{*}\right)$ and $e \in E$, we will denote by $|\mu|$ the variation of $\mu$ and by $\langle e, \mu\rangle$ the element of $M(X)$ defined on each Borel subset $B$ of $X$ by

$$
\langle e, \mu\rangle(B)=\mu(B)(e) .
$$

The duality between $M\left(X, E^{*}\right)$ and $C(X, E)$ is then defined as follows: for each $f \in C(X)$ and $e \in E$,

$$
\mu(f \otimes e)=\int_{X} f d\langle e, \mu\rangle,
$$

where $f \otimes e$ is the element of $C(X, E)$ defined by

$$
f \otimes e(x)=f(x) e, \text { for all } x \in X .
$$

If $B$ is a Borel subset of $X$, then $1_{B}$ will denote the characteristic function of $B$, and if $e \in E$ we let $1_{B} \otimes e$ denote the element of $C(X, E)^{* *}$ defined by

$$
1_{B} \otimes e(\mu)=\langle e, \mu\rangle(B)=\mu(B)(e),
$$

for each $\mu \in M\left(X, E^{*}\right)$.

If $X$ is a compact Hausdorff space, $E$ and $F$ are Banach spaces, every bounded linear operator $T: C(X, E) \rightarrow F$ has a representing measure $G$. The measure $G$ is defined on the $\sigma$-field $\Sigma$ of Borel subsets of $X$ and takes its values in $\mathscr{L}\left(E, F^{* *}\right)$, the space of all bounded linear operators from $E$ to $F^{* *}$. The measure $G$ is such that, for each Borel subset $B$ of $X$ and for each $e \in E$,

$$
G(B) e=T^{* *}\left(1_{B} \otimes e\right) .
$$

For $y^{*} \in F^{*}$, if we denote by $G_{y^{*}}$ the $E^{*}$-valued measure on $X$ such that, for each Borel subset $B$ of $X$ and each $e \in E$,

$$
\left\langle e, G_{y^{*}}\right\rangle(B)=\left\langle y^{*}, G(B) e\right\rangle,
$$

then $G_{y^{*}}$ is the unique element of $M\left(X, E^{*}\right)$ that represents $T^{*} y^{*}$ in the sense that, for each $f \in C(X, E)$,

$$
\left\langle y^{*}, T f\right\rangle=\int_{X} f(x) d G_{y^{*}}(x) .
$$

If $E$ and $F$ are Banach spaces, we denote by $E \otimes_{\varepsilon} F$ the algebraic tensor product of $E$ and $F$ endowed with the norm $\|\cdot\|_{\varepsilon}$ :

$$
\left\|\sum_{i=1}^{m} x_{i} \otimes y_{i}\right\|_{\varepsilon}=\sup \left\{\left|\sum_{i=1}^{m} x^{*}\left(x_{i}\right) y^{*}\left(y_{i}\right)\right|:\left\|x^{*}\right\|,\left\|y^{*}\right\| \leq 1\right\} .
$$

The completion $E \widehat{\otimes}_{\varepsilon} F$ of $E \otimes_{\varepsilon} F$ is called the injective tensor product of $E$ and $F$. 
A bounded linear operator $T: E \rightarrow F$ from a Banach space $E$ into a Banach space $F$ is said to be an integral operator if the bilinear form $\tau$ on $E \times F^{*}$ defined by $\tau\left(e, y^{*}\right)=y^{*}(T e)$ for $e \in E$ and $y^{*} \in F^{*}$ determines an element of $\left(E \widehat{\otimes}_{\varepsilon} F^{*}\right)^{*}$, the dual of the injective tensor product $E \widehat{\otimes}_{\varepsilon} F^{*}$ of the Banach spaces $E$ and $F^{*}$. The integral norm of $T$, which we will denote by $\|T\|_{\text {int }}$, is just the norm of the bounded linear functional induced by $\tau$ as an element of the dual space $\left(E \widehat{\otimes}_{\varepsilon} F^{*}\right)^{*}$. Hence, if $T: E \rightarrow F$ is an integral operator, then

$$
\|T\|_{\text {int }}=\sup \left\{\left|\sum_{i=1}^{n} y_{i}^{*}\left(T e_{i}\right)\right|:\left\|\sum_{i=1}^{n} e_{i} \otimes y_{i}^{*}\right\|_{\varepsilon} \leq 1\right\} .
$$

Finally, $I(E, F)$ will stand for the Banach space of all integral operators from $E$ to $F$ equipped with the integral norm. For all undefined notions and notations we refer the reader to [3], [4], or [5].

One of the most useful tools in the study of an integral operator $T$ between two Banach spaces $E$ and $F$ is its representation by a finite regular Borel scalar measure on the compact $B\left(E^{*}\right) \times B\left(F^{* *}\right)$, the product of the closed unit balls of $E^{*}$ and $F^{* *}$ equipped with their weak ${ }^{*}$-topologies. This, of course, goes back to Grothendieck [6] and can be used to characterize integral operators by the following, which we will state and prove before proving the main result:

Proposition 1. A bounded linear operator $T: E \rightarrow F$ between two Banach spaces is an integral operator if and only if there exists a regular $F^{* *}$-valued vector measure $m$ of bounded variation defined on the $\sigma$-field of Borel subsets of the closed unit ball $B\left(E^{*}\right)$, such that, for each $e \in E$,

$$
T e=\int_{B\left(E^{*}\right)} e^{*}(e) d m\left(e^{*}\right) .
$$

In this case $m$ can be chosen so that $\|T\|_{\text {int }}=|m|\left(B\left(E^{*}\right)\right)$.

Proof. Assume that there exists a regular $F^{* *}$-valued vector measure $m$ defined on the $\sigma$-field of Borel subsets of $B\left(E^{*}\right)$ with $|m|\left(B\left(E^{*}\right)\right)<\infty$ such that, for each $e \in E$,

$$
T e=\int_{B\left(E^{*}\right)} e^{*}(e) d m\left(e^{*}\right) .
$$

This in particular shows that the operator $J \circ T: E \rightarrow F^{* *}$, where $J$ denotes the natural embedding of $F$ into $F^{* *}$, has an extension $\widehat{T}$ to an integral operator from $C\left(B\left(E^{*}\right)\right)$ to $F^{* *}$. Hence $T$ is an integral operator [4, p. 233], and

$$
\|T\|_{\text {int }} \leq\|\widehat{T}\|_{\text {int }}=|m|\left(B\left(E^{*}\right)\right) \text {. }
$$

Conversely, suppose that $T: E \rightarrow F$ is an integral operator; then it follows from [4, page 231] that there exists a regular Borel measure $\mu$ on $B\left(E^{*}\right) \times$ $B\left(F^{* *}\right)$ such that, for each $e \in E$ and $y^{*} \in F^{*}$,

$$
\left\langle y^{*}, T e\right\rangle=\int_{B\left(E^{*}\right) \times B\left(F^{* *}\right)} e^{*}(e) y^{* *}\left(y^{*}\right) d \mu\left(e^{*}, y^{* *}\right)
$$


and

$$
\|T\|_{\text {int }}=|\mu|\left(B\left(E^{*}\right) \times B\left(F^{* *}\right)\right) .
$$

Following [4, p. 234], define

$$
S: E \rightarrow L_{\infty}(\mu) \quad \text { by } \quad \operatorname{Se}\left(e^{*}, y^{* *}\right)=e^{*}(e)
$$

and

$$
R: F^{*} \rightarrow L_{\infty}(\mu) \quad \text { by } \quad R y^{*}\left(e^{*}, y^{* *}\right)=y^{* *}\left(y^{*}\right) .
$$

Then $S$ and $R$ are bounded linear operators with $\|S\|,\|R\| \leq 1$. Let $Q$ be the restriction of $R^{*}$ to $L_{1}(\mu)$; then $Q$ is a bounded linear operator from $L_{1}(\mu)$ into $F^{* *}$. It is immediate that $J \circ T=Q \circ I \circ S$ where $I: L_{\infty}(\mu) \rightarrow L_{1}(\mu)$ is the natural inclusion and $J: F \rightarrow F^{* *}$ is the natural embedding. This in particular shows that the operator $Q \circ I$ restricted to $C\left(B\left(E^{*}\right) \times B\left(F^{* *}\right)\right)$ is an integral operator whose representing $F^{* *}$-valued measure $\hat{\mu}$ is such that

$$
\hat{\mu}(C)=Q\left(1_{C}\right)
$$

for each Borel subset $C$ of $B\left(E^{*}\right) \times B\left(F^{* *}\right)$. Finally, note that since the natural projection of $B\left(E^{*}\right) \times B\left(F^{* *}\right)$ onto $B\left(E^{*}\right)$ is a continuous mapping, it induces a bounded linear operator from $C\left(B\left(E^{*}\right)\right)$ into $C\left(B\left(E^{*}\right) \times B\left(F^{* *}\right)\right)$ as follows: for $\phi \in C\left(B\left(E^{*}\right)\right)$, let $\hat{\phi} \in C\left(B\left(E^{*}\right) \times B\left(F^{* *}\right)\right)$ be such that

$$
\hat{\phi}\left(e^{*}, y^{* *}\right)=\phi\left(e^{*}\right) \text {. }
$$

This, of course, shows that the operator $J \circ T$ extends to an integral operator

$$
\widehat{T}: C\left(B\left(E^{*}\right)\right) \rightarrow F^{* *}
$$

such that, for each $\phi \in C\left(B\left(E^{*}\right)\right)$,

$$
\widehat{T} \phi=Q \circ I(\hat{\phi}) \text {. }
$$

It is immediate that the $F^{* *}$-valued measure $m$ representing $\widehat{T}$ is such that

$$
m(B)=Q\left(1_{B \times B\left(F^{* *}\right)}\right)
$$

and

$$
|m|\left(B\left(E^{*}\right) \leq|\mu|\left(B\left(E^{*}\right) \times B\left(F^{* *}\right)\right)=\|T\|_{\text {int }} .\right.
$$

Since $\widehat{T}$ extends $T$, it follows that

$$
\|T\|_{\text {int }}=|m|\left(B\left(E^{*}\right)\right) .
$$

\section{MAIN Result}

Throughout this section, $X$ is a compact Hausdorff space, $E$ and $F$ are Banach spaces, and $T: C(X, E) \rightarrow F$ is a bounded linear operator with representing measure $G$. The main result of this paper gives a characterization of integral operators $T$ in terms of some properties of $G$. The first step to achieve such a characterization is to show that, if $T$ is an integral operator on $C(X, E)$, then one can do a little better than Proposition 1 by representing the 
operator $T$ by a regular $F^{* *}$-valued measure of bounded variation defined on the $\sigma$-field of Borel subsets of $X \times B\left(E^{*}\right)$ rather than on the whole unit ball of $C(X, E))^{*}$. The proof we present here is different from our earlier proof, which relied on our result [7]. We would like to thank the referee for suggesting the following approach, which relies more on basic knowledge and classical results of vector measures that can be found in [4] or [5].

Lemma 2. If $T: C(X, E) \rightarrow F$ is an integral operator, then there exists a regular $F^{* *}$-valued measure of bounded variation $\theta$ on the $\sigma$-field of Borel subsets of $X \times B\left(E^{*}\right)$, such that for each $f \in C(X, E)$ and $y^{*} \in F^{*}$,

$$
\left\langle y^{*}, T f\right\rangle=\int_{X \times B\left(E^{*}\right)} e^{*}(f(x)) d \theta\left(x, e^{*}\right)
$$

and

$$
\|T\|_{\text {int }}=|\theta|\left(X \times B\left(E^{*}\right)\right) .
$$

Proof. Suppose that $T: C(X, E) \rightarrow F$ is an integral operator, then the bilinear map $\tau\left(f, y^{*}\right)=y^{*}(T(f))$ for $f \in C(X, E)$ and $y^{*} \in F^{*}$ defines an element of $\left(C(X, E) \otimes_{\varepsilon} F^{*}\right)^{*}$. It is easy to check that $C(X, E) \otimes_{\varepsilon} F^{*}$ embeds isometrically in $C\left(X \times B\left(E^{*}\right) \times B\left(F^{* *}\right)\right)$ such that, if $f \in C(X, E)$ and $y^{*} \in F^{*}$,

$$
f \otimes y^{*}\left(x, e^{*}, y^{* *}\right)=e^{*}(f(x)) y^{* *}\left(y^{*}\right)
$$

for each $\left(x, e^{*}, y^{* *}\right) \in \Omega=X \times B\left(E^{*}\right) \times B\left(F^{* *}\right)$. Hence, by the Hahn-Banach theorem, there exists a regular Borel measure $\mu$ on $\Omega$ so that, for each $f \in$ $C(X, E)$ and $y^{*} \in F^{*}$,

$$
\left\langle y^{*}, T f\right\rangle=\int_{\Omega} e^{*}(f(x)) y^{* *}\left(y^{*}\right) d \mu\left(x, e^{*}, y^{* *}\right)
$$

and

$$
\|T\|_{\text {int }}=|\mu|(\Omega) .
$$

The proof now follows the steps of Proposition 1. Indeed, since the natural projection of $\Omega$ onto $X \times B\left(E^{*}\right)$ is continuous, it induces a bounded linear operator

$$
S: C\left(X \times B\left(E^{*}\right)\right) \rightarrow L_{\infty}(\mu)
$$

such that, if $\phi \in C\left(X \times B\left(E^{*}\right)\right)$, then

$$
S(\phi)\left(x, e^{*}, y^{* *}\right)=\phi\left(x, e^{*}\right)
$$

for all $\left(x, e^{*}, y^{* *}\right) \in \Omega$.

Let $R: F^{*} \rightarrow L_{\infty}(\mu)$ be defined by $R\left(y^{*}\right)\left(x, e^{*}, y^{* *}\right)=y^{* *}\left(y^{*}\right)$, and let $Q$ be the restriction of $R^{*}$ to $L_{1}(\mu)$. It is straightforward to check that the operator $Q \circ I \circ S$ is an integral operator whose representing measure $\theta$ is such that

$$
\theta(C)=Q\left(1_{C \times B\left(F^{* *}\right)}\right)
$$

for each Borel subset $C$ of $X \times B\left(E^{*}\right)$, and

$$
|\theta|\left(X \times B\left(E^{*}\right)\right) \leq|\mu|(\Omega)=\|T\|_{\text {int }} .
$$


Moreover, note that $C(X, E)$ embeds isometrically in $C\left(X \times B\left(E^{*}\right)\right)$ by

$$
f\left(x, e^{*}\right)=e^{*}(f(x))
$$

for each $f \in C(X, E)$ and $\left(x, e^{*}\right) \in X \times B\left(E^{*}\right)$. It is easy to see that, for each $f \in C(X, E)$,

$$
J \circ T(f)=Q \circ I \circ S(f) ;
$$

hence

and

$$
T(f)=\int_{X \times B\left(E^{*}\right)} e^{*}(f(x)) d \theta\left(x, e^{*}\right)
$$

$$
\|T\|_{\text {int }} \leq\|Q \circ I \circ S\|_{\text {int }} \leq|\theta|\left(X \times B\left(E^{*}\right)\right) .
$$

This complete the proof of Lemma 2.

We are now ready to state and prove the main result of this paper.

Theorem 3. Let $X$ be a compact Hausdorff space, let $E$ and $F$ be Banach spaces, and let $T: C(X, E) \rightarrow F$ be a bounded linear operator with representing measure $G$. Then $T$ is an integral operator if and only if, for each Borel subset $B$ of $X$, the operator $G(B): E \rightarrow F$ is integral and the measure $G$ is of finite variation as a vector measure taking its values in $I(E, F)$, which is equipped with the integral norm.

Proof. Assume that $T: C(X, E) \rightarrow F$ is an integral operator. Let $G$ denote the vector measure representing the operator $T$; hence, for each Borel subset $B$ of $X$ and each $e \in E$,

$$
G(B) e=T^{* *}\left(1_{B} \otimes e\right) .
$$

It is clear at this stage that, since $T^{* *}$ is also integral [4, p. 236] then, for each Borel subset $B$ of $X$, the operator $G(B): E \rightarrow F$ is an integral operator since it is the composition of $T^{* *}$ and the bounded linear operator $E \rightarrow C(X, E)^{* *}$ which to each $e$ in $E$ associates the element $1_{B} \otimes e$. In what follows we shall concentrate on estimating the value of $\|G(B)\|_{\text {int }}$. For this, note that by Lemma 2 there exists a regular $F^{* *}$-valued measure $\theta$ defined on the $\sigma$-field of Borel subsets of $X \times B\left(E^{*}\right)$ such that, for each $f \in C(X, E)$,

$$
T(f)=\int_{X \times B\left(E^{*}\right)} e^{*}(f(x)) d \theta\left(x, e^{*}\right)
$$

and

$$
\|T\|_{\text {int }}=|\theta|\left(X \times B\left(E^{*}\right)\right) .
$$

It follows that, for each $y^{*} \in F^{*}$ and $f \in C(X, E)$,

$$
\left\langle T^{*} y^{*}, f\right\rangle=\int_{X \times B\left(E^{*}\right)} e^{*}(f(x)) d \theta_{y^{*}}\left(x, e^{*}\right),
$$

where $\theta_{y^{*}}$ is the scalar measure on $X \times B\left(E^{*}\right)$ defined by

$$
\theta_{y^{*}}(C)=\theta(C)\left(y^{*}\right)
$$

for each Borel subset $C$ of $X \times B\left(E^{*}\right)$. 
We claim that, for each Borel subset $B$ of $X$ and each $e \in E$,

$$
G(B) e=\int_{B \times B\left(E^{*}\right)} e^{*}(e) d \theta\left(x, e^{*}\right) .
$$

For this, suppose that $y^{*} \in F^{*}$ and $K$ is a compact $G_{\delta}$ subset of $X$. Let $\left(u_{n}\right)_{n \geq 1}$ be a sequence of continuous real-valued functions so that $0 \leq u_{n} \leq 1$ for all $n \geq 1$ and $u_{n}$ converges to $1_{K}$ pointwise. If $e \in E$, then

$$
\begin{aligned}
\left\langle G(K) e, y^{*}\right\rangle & =\left\langle e, G_{y^{*}}\right\rangle(K) \\
& =\lim _{n \rightarrow \infty}\left\langle y^{*}, T\left(u_{n} \otimes e\right)\right\rangle \\
& =\lim _{n \rightarrow \infty} \int_{X \times B\left(E^{*}\right)} u_{n}(x) e^{*}(e) d \theta_{y^{*}}\left(x, e^{*}\right) \\
& =\int_{J_{X \times B\left(E^{*}\right)}} 1_{K}(x) e^{*}(e) d \theta_{y^{*}}\left(x, e^{*}\right) \\
& =\int_{K \times B\left(E^{*}\right)} e^{*}(e) d \theta_{y^{*}}\left(x, e^{*}\right) .
\end{aligned}
$$

Therefore,

$$
G(K) e=\int_{K \times B\left(E^{*}\right)} e^{*}(e) d \theta\left(x, e^{*}\right) .
$$

Moreover, since for each $y^{*} \in F^{*}$ the $E^{*}$-valued vector measure $G_{y^{*}}$ is regular, it follows that

$$
G(B) e=\int_{B \times B\left(E^{*}\right)} e^{*}(e) d \theta\left(x, e^{*}\right)
$$

for all Borel subsets $B$ of $X$. This proves our claim. If we denote by $p$ : $X \times B\left(E^{*}\right) \rightarrow E^{*}$ the projection mapping which to each $\left(x, e^{*}\right)$ in $X \times B\left(E^{*}\right)$ associates $e^{*}$ in $B\left(E^{*}\right)$, then for each Borel subset $B$ of $X$, let $\lambda_{B}$ denote the regular $F^{* *}$-valued measure defined on the $\sigma$-field of Borel subsets of $B\left(E^{*}\right)$ as follows: for each Borel subset $V$ of $B\left(E^{*}\right)$,

$$
\lambda_{B}(V)=\theta(B \times V) \text {. }
$$

In other words, $\lambda_{B}=\theta \mid B \times B\left(E^{*}\right) \circ p^{-1}$, which is the image measure of the restriction of $\theta$ to $B \times B\left(E^{*}\right)$ by $p$. This implies that, for each $e \in E$,

$$
\int_{B\left(E^{*}\right)} e^{*}(e) d \lambda_{B}\left(e^{*}\right)=\int_{B \times B\left(E^{*}\right)} e^{*}(e) d \theta\left(x, e^{*}\right) .
$$

Equations $(*)$ and $(* *)$ show that the measure $\lambda_{B}$ is a regular, $F^{* *}$-valued measure that represents the operator $G(B): E \rightarrow F$. Hence, by Proposition 1,

$$
\|G(B)\|_{\text {int }} \leq\left|\lambda_{B}\right|\left(B\left(E^{*}\right)\right)=|\theta|\left(B \times B\left(E^{*}\right)\right),
$$

and therefore

$$
\|G(B)\|_{\text {int }} \leq|\theta|\left(B \times B\left(E^{*}\right)\right) .
$$


Hence the vector measure $G$ representing the operator $T$ takes its values in $I(E, F)$, and it follows from $(\dagger)$ that

$$
|G|_{\text {int }}(X) \leq|\theta|\left(X \times B\left(E^{*}\right)\right) .
$$

Here, of course, $|G|_{\text {int }}(X)=\sup \sum_{B_{i} \in \pi}\left\|G\left(B_{i}\right)\right\|_{\text {int }}$, where the sup is taken over all the finite partitions of $X$ into Borel subsets of $X$.

Conversely, suppose $T: C(X, E) \rightarrow F$ is such that

$$
G: \sum \rightarrow I(E, F) \text {, and }|G|_{\text {int }}(X)<\infty ;
$$

we need to show that $T$ is an integral operator. For this, note that if $J$ denotes the natural embedding of $F$ into $F^{* *}$, then by [4, p. 233] it is enough to show that $J \circ T: C(X, E) \rightarrow F^{* *}$ is an integral operator. The mapping $J$ induces a mapping $\widehat{J}: I(E, F) \rightarrow I\left(E, F^{* *}\right)$ defined as follows, for each $U \in I(E, F)$ :

$$
\widehat{J}(U)=J \circ U
$$

this in turn induces a vector measure $\widehat{G}: \sum \rightarrow I\left(E, F^{* *}\right)$ such that

$$
\widehat{G}(B)=\widehat{J}(G(B)),
$$

for all $B \in \sum$. It is immediate that $\widehat{G}$ is the measure representing the operator $J \circ T$. Since $|G|_{\text {int }}(X)<\infty$, it follows easily that $|\widehat{G}|_{\text {int }}(X)<\infty$. Moreover, since $I\left(E, F^{* *}\right)$ is isometric to the dual space of the injective tensor product $E \widehat{\otimes}_{\varepsilon} F^{*}[4$, p. 237], it follows that $\widehat{G}$ is a vector measure of bounded variation taking its values in the dual space $\left(E \widehat{\otimes}_{\varepsilon} F^{*}\right)^{*}$. Since $|\widehat{G}|_{\text {int }}(X)<\infty$, it follows from $[4$, p. 7] that $\widehat{G}$ is strongly additive. Recall that, for each $e \in E$ and $y^{*} \in F^{*}$, the scalar measure $\left\langle e, G_{y^{*}}\right\rangle$ is in $M(X)$. Hence, for $u=\sum_{i=1}^{n} e_{i} \otimes$ $y_{i}^{*} \in E \widehat{\otimes}_{\varepsilon} F^{*}$, let $\langle u, \widehat{G}\rangle$ be the scalar measure defined on $X$ as follows: for each Borel subset $B$ of $X$,

$$
\langle u, \widehat{G}\rangle(B)=\widehat{G}(B)(u)=\sum_{i=1}^{n}\left\langle G(B) e_{i}, y_{i}^{*}\right\rangle=\sum_{i=1}^{n}\left\langle e_{i}, G_{y_{i}^{*}}\right\rangle(B) .
$$

This implies that for each $u \in E \widehat{\otimes}_{\varepsilon} F^{*}$, the measure $\langle u, \widehat{G}\rangle \in M(X)$. This in particular shows that $\widehat{G}: \sum \rightarrow\left(E \widehat{\otimes}_{\varepsilon} F^{*}\right)^{*}$ is countably additive with respect to the weak ${ }^{*}$ topology on $\left(E \widehat{\otimes}_{\varepsilon} F^{*}\right)^{*}$. Since $\widehat{G}$ is strongly additive, it follows easily that $G$ is countably additive. Finally, since $\widehat{G}$ is weak ${ }^{*}$ regular, a glance at $[4$, p. 157] reveals that it is also regular. It follows that $\widehat{G}$ defines an element of $C\left(X, E \widehat{\otimes}_{\varepsilon} F^{*}\right)^{*}$; hence, by [5, p. 269], there exists a weak ${ }^{*}|\widehat{G}|_{\text {int }}$-integrable function

$$
h: X \rightarrow I\left(E, F^{* *}\right) \simeq\left(E \widehat{\otimes}_{\varepsilon} F^{*}\right)^{*}
$$

such that $\|h(x)\|_{\text {int }}=1|\widehat{G}|_{\text {int }}$ a.e., $\widehat{G}=h|\widehat{G}|_{\text {int }}$ and, for each $f \in C\left(X, E \widehat{\otimes}_{\varepsilon} F^{*}\right)$,

$$
\langle f, \widehat{G}\rangle=\int_{X}\langle f(x), h(x)\rangle d|\widehat{G}|_{\mathrm{int}}(x) .
$$


In particular, if $\varphi \in C(X, E)$ and $y^{*} \in F^{*}$, let $f=\varphi \otimes y^{*}$ be the element of $C\left(X, E \widehat{\otimes}_{\varepsilon} F^{*}\right)$ defined by

$$
\varphi \otimes y^{*}(x)=\varphi(x) \otimes y^{*} \text { for all } x \in X,
$$

then

$$
\left\langle\varphi \otimes y^{*}, \widehat{G}\right\rangle=\int_{X} y^{*}\langle h(x), \varphi(x)\rangle d|\widehat{G}|_{\mathrm{int}} \mid(x) .
$$

But we also have

$$
\left\langle J \circ T \varphi, y^{*}\right\rangle=\left\langle\varphi, \widehat{G}_{y^{*}}\right\rangle,
$$

where $\widehat{G}_{y^{*}}$ is the element of $M\left(X, E^{*}\right)$ such that, for each Borel subset $B$ of $X$,

$$
\widehat{G}_{y^{*}}(B)=y^{*}(\widehat{G}(B))=y^{*}(G(B)) .
$$

Hence since $\widehat{G}=h \cdot|\widehat{G}|_{\text {int }}$, it follows that for each Borel subset $B$ of $X$,

$$
\widehat{G}_{y^{*}}(B)=\int_{B} y^{*} h(x) d|\widehat{G}|_{\text {int }}(x) .
$$

This implies that, for each $\varphi \in C(X, E)$,

$(* * * *)$

$$
\left\langle\varphi, \widehat{G}_{y^{*}}\right\rangle=\int_{X} y^{*}\langle h(x), \varphi(x)\rangle d|\widehat{G}|_{\text {int }}(x) .
$$

It follows that if $\sum_{i=1}^{n} \varphi_{i} \otimes y_{i}^{*}$ is in $C(X, E) \otimes_{\varepsilon} F^{*}$ with $\left\|\sum_{i=1}^{n} \varphi_{i} \otimes y_{i}^{*}\right\|_{\varepsilon} \leq 1$, then, for each $x$ in $X$,

$$
\left|\left\langle h(x), \sum_{i=1}^{n} \varphi_{i}(x) \otimes y_{i}^{*}\right\rangle\right| \leq\|h(x)\|_{\mathrm{int}},
$$

since

$$
\left\|\sum_{i=1}^{n} \varphi_{i}(x) \otimes y_{i}^{*}\right\|_{\varepsilon} \leq\left\|\sum_{i=1}^{n} \varphi_{i} \otimes y_{i}^{*}\right\|_{\varepsilon} \leq 1 .
$$

It follows then, from $(* * *)$ and $(* * * *)$, that

$$
\left\|\sum_{i=1}^{n}\left\langle T \varphi_{i}, y_{i}^{*}\right\rangle\right\| \leq|\widehat{G}|_{\text {int }}(X)<\infty .
$$

Hence $J \circ T$ is an element of $I\left(C(X, E), F^{* *}\right)$, which implies that $T \in$ $I(C(X, E), F)$. This completes the proof.

\section{APPLICATIONS}

The study of integral operators on $C(X, E)$ spaces was motivated by some problems that arose in [8] concerning nuclear operators on $C(X, E)$ spaces. Recall that an operator $T$ between two Banach spaces $Y$ and $Z$ is said to be a nuclear operator if there exist sequences $\left(y_{n}^{*}\right)$ and $\left(z_{n}\right)$ in $Y^{*}$ and $Z$, respectively, such that $\sum_{n}\left\|y_{n}^{*}\right\|\left\|z_{n}\right\|<\infty$ and, for each $y \in Y$,

$$
T y=\sum_{n} y_{n}^{*}(y) z_{n} \text {. }
$$


The nuclear norm is defined by

$$
\|T\|_{\text {nuc }}=\inf \left\{\sum_{n}\left\|y_{n}^{*}\right\|\left\|z_{n}\right\|\right\},
$$

where the infimum is taken over all sequences $\left(y_{n}^{*}\right)$ and $\left(z_{n}\right)$ such that $T y=$ $\sum_{n} y_{n}^{*}(y) z_{n}$ for all $y \in Y$. We shall denote by $N(Y, Z)$ the space of nuclear operators from $Y$ to $Z$ under the nuclear norm. The study of nuclear operators on $C(X, E)$ spaces was initiated in [1], where some of the known results in the scalar case were extended. Nuclear operators on $C(X, E)$ spaces were also considered in [2], where it was shown that if $T: C(X, E) \rightarrow F$ is a nuclear operator, then for each Borel subset $B$ of $X$, the operator $G(B): E \rightarrow F$ is nuclear. In [8] it was shown that the representing measure $G$ of a nuclear operator is in fact of bounded variation as a vector measure taking its values in $N(E, F)$. Hence all the above known results can be summarized as follows:

Proposition 4. Let $X$ be a compact Hausdorff space, and let $E$ and $F$ be two Banach spaces. If $T$ is a nuclear operator from $C(X, E)$ into $F$ with representing measure $G$, then

(i) For any Borel subset $B$ of $X$, the operator $G(B): E \rightarrow F$ is nuclear, and

(ii) $G$ is of bounded variation as a vector measure taking its values in $N(E, F)$ under the nuclear norm.

Easy examples show that conditions (i) and (ii) above do not characterize nuclear operators on $C(X, E)$ spaces. As a matter of fact, it was shown in [8] that counterexamples can be given as soon as the range space $F$ fails to have the so-called Radon-Nikodym property (RNP) [4]. The interesting question that arises is then the following:

Question 5. Assuming that $F$ has the (RNP) and that $T: C(X, E) \rightarrow F$ is an operator whose representing measure $G$ satisfies conditions (i) and (ii) of Proposition 4 , is $T$ a nuclear operator?

In what follows, we shall show how Theorem 3 can be used to give a positive answer to Question 5 in case $F$ is assumed to be complemented in its bidual.

Theorem 6. Let $F$ be a Banach space with the Radon-Nikodym property, and assume that $F$ is complemented in its bidual. A bounded linear operator $T$ : $C(X, E) \rightarrow F$ is nuclear whenever its representing vector measure $G$ satisfies the following conditions:

(i) For each Borel subset $B$ of $X$, the operator $G(B): E \rightarrow F$ is nuclear, and

(ii) $G$ is of bounded variation as a vector measure taking its values in $N(E, F)$.

Proof. First note that, for an arbitrary Banach space $F$, any element $U$ in $N(E, F)$ is in $I(E, F)$ with $\|U\|_{\text {int }} \leq\|U\|_{\text {nuc }}$. Hence, by Theorem 3, an 
operator $T: C(X, E) \rightarrow F$ that satisfies conditions (i) and (ii) above is indeed integral. Therefore, when in addition we assume that $F$ is complemented in its bidual $F^{* *}$, the operator $T$ becomes Pietsch integral (also called strictly integral) $[4$, p. 235]. Under these conditions, and if $F$ is assumed to have the Radon-Nikodym property, it is well known [4, p. 175] that $T$ becomes a nuclear operator. This completes the proof.

Remark. As we have just seen, Theorem 6 is a direct application of the main result of this paper, it should be noted that Theorem 6 gives a positive answer to Question 5 when $F$ is a dual space and when $F$ is a Banach lattice.

\section{BIBLIOGRAPHY}

1. G. Alexander, Linear operators on the space of vector-valued continuous functions, Ph.D. thesis, New Mexico State University, Las Cruces, New Mexico, 1972.

2. R. Bilyeu and P. Lewis, Some mapping properties of representing measures, Ann. Math. Pure Appl. 109 (1976), 273-287.

3. G. Choquet, Lecture on analysis, Vol. II, Benjamin, New York, 1969.

4. J. Diestel and J. J. Uhl, Jr., Vector measures, Math. Surveys 15 Amer. Math. Soc., Providence, RI, 1977.

5. N. Dinculeanu, Vector measures, Pergamon, Press, 1967.

6. A. Grothendieck, Produits tensoriels topologiques et espaces nucléaires, Memoirs Amer. Math. Soc. 16 (1955), 1-140.

7. P. Saab, Integral representation by boundary vector measures, Canad. Math. Bull. 25 (1982), 164-168.

8. B. Smith, Some bounded linear operators on the spaces $C(\Omega, E)$ and $A(K, E)$, Ph.D. thesis, University of Missouri, Columbia, MO, 1989.

9. C. Swartz, Absolutely summing and dominated operators on spaces of vector valued continuous functions, Trans. Amer. Math. Soc. 179 (1973), 123-132.

Department of Mathematics, University of Missouri, Columbia, Missouri 65211 